\title{
Les dictionnaires de poche français-italien et italien-français $(1710-2005)^{1}$
}

\author{
Jacqueline Lillo \\ Université de Palerme \\ jlillo@unipa.it
}

\section{Introduction, définition}

\section{1}

Les termes de poche ou de tascabile ont plusieurs synonymes, en français : portatif, abrégé, petit, en italien: portatile, compendiato, piccolo, compatto. L'utilisation de ces mots dépend de la date de publication des ouvrages. Aux XVIII-XIX ${ }^{\mathrm{e}}$ siècles, alors que le français hésite entre portatif et de poche, l'italien préfère portatile. Le terme italien moderne tascabile (qui signifie «qui peut être mis dans la poche ») est postérieur et ne se trouve pas dans les premiers ouvrages. Piccolo (petit) est un terme du $\mathrm{XX}^{\mathrm{e}}$ siècle (Piccolo Mariotti, Piccolo Ghiotti, ...) ; ces derniers sont inclus dans notre étude parce que les auteurs eux-mêmes les présentent comme des «poches ».

\section{2}

Si nous observons avec attention ces ouvrages, force est de constater que les critères de définition des «portatifs» ou "poche» changent avec les années. Aux XVIII ${ }^{\mathrm{e}}-\mathrm{XIX}{ }^{\mathrm{e}}$ siècles, cela signifie seulement que le format est réduit par rapport à un dictionnaire précédent avec lequel on le compare, ou par rapport aux dimensions des dictionnaires en circulation. Ainsi, l'Alberti ${ }^{2}$ portatif de 1775 (Venise, Bettinelli) est en deux volumes in- $8^{\circ}$, de 908 et 1087 pages, et mesure $13,5 \mathrm{~cm} \times 18,5 \mathrm{~cm}$ pour respectivement $6,2 \mathrm{~cm}$ et $7 \mathrm{~cm}$ d'épaisseur. Il est plus petit de toute façon que les autres Alberti, le plus souvent publiés en in- $4^{\circ}$. De même, les Cormon ${ }^{3}$ et Manni ${ }^{4}$ in $-8^{\circ}$ du début du XIX ${ }^{\mathrm{e}}$ sont des portatifs même si leurs dimensions, bien inférieures à celles des Alberti in- $4^{\circ}$ alors extrêmement diffusés, sont celles d'un dictionnaire format entier d'aujourd'hui (Cormon et Manni, 1807, Lyon, Blanc : 13,5 cm $\times 21 \mathrm{~cm} \times 5,2 \mathrm{~cm}$ ). A côté de ces dictionnaires, existent les Briccolani, Barberi, Ronna, Ruggieri $(7,5 \mathrm{~cm} \times 11,5 \mathrm{~cm} \times 4,5 \mathrm{~cm})$, etc., dont le format est presque identique à celui des Collins Mondadori de la fin du $\mathrm{XX}^{\mathrm{e}}$. Aux $\mathrm{XX}^{\mathrm{e}}$ et $\mathrm{XXI}^{\mathrm{e}}$ siècles, on note une extrême diversité de dimensions. On passe de la Collection « Poucet » chez Hatier $(4 \mathrm{~cm} \times 6,3$ $\mathrm{cm} \times 2 \mathrm{~cm})$ ou «Mignon » chez Bietti $\left(3,5 \mathrm{~cm} \times 5 \mathrm{~cm} \times 1,3 \mathrm{~cm}\right.$, in $\left.-32^{\circ}\right)$ au «Dictionnaire de Poche » de Larousse, au Livre de Poche (Garzanti, Librairie Générale Française), au Robert-Collins Poche italien (2005), tous, à quelques millimètres près, de 10,6 cm de large pour 17,6 cm de long et une épaisseur de 3 ou $4 \mathrm{~cm}$. Enfin, certains dictionnaires sont de toute évidence des « poches » à cause de leurs dimensions mais ne le signalent nulle part (Moussard, Garrus 1982, ou encore Anonyme 1995, chez Polaris, avec $10,5 \mathrm{~cm} \times 14,5 \mathrm{~cm} \times 2,3 \mathrm{~cm}$ pour ce dernier, par exemple).

\section{3}

Les tout premiers dictionnaires bilingues : Giovanni Antonio Fenice (1584, Du Puys, Morges et Paris : $10 \mathrm{~cm} \times 16 \mathrm{~cm} \times 3,5 \mathrm{~cm})$, Pierre $\operatorname{Canal}^{5}(1598,1603$, Genève, Chouet : $10,5 \mathrm{~cm} \times 16,5 \mathrm{~cm} \times 3,5 \mathrm{~cm})$, Filippo Venuti (1614, Cologny, Chouet: $11 \mathrm{~cm} \times 18 \mathrm{~cm} \times 7 \mathrm{~cm})$ ont un format poche que les dictionnaires suivants n'adoptent pas. Ils servent sans doute aux négociants et voyageurs. Nous ne les avons pas inclus dans la catégorie des poches car il est impossible de les définir par comparaison avec un format plus grand qui n'existe pas à l'époque, tout au moins pour les bilingues français / italien. En revanche, l'ouvrage La Reggia di Mercurio d'Anguselli da Lucca, (Venise, Pavin), divisé en différents « appartements » (comme l'auteur appelle ses différents dictionnaires bilingues ${ }^{6}$ ), peut être considéré 
comme un dictionnaire de poche $(11,8 \mathrm{~cm} \times 17,8 \mathrm{~cm} \times 2,4 \mathrm{~cm})$ car il sort à une date $(1710)$ de grande diffusion des Veneroni ${ }^{7}$, qui adoptent des dimensions $(22 \mathrm{~cm} \times 30 \mathrm{~cm} \times 7 \mathrm{~cm}$ pour l'édition de 1710 , Paris, De Lormes) plus proches des dictionnaires plein format d'aujourd'hui.

\section{Données numériques et diffusion de ces dictionnaires}

\section{1}

Dans l'état actuel de nos recherches (Lillo éd. 2007), 283 dictionnaires de poche italien / français ont pu être dénombrés de 1710 à 2005 (voir Appendice). Onze dictionnaires seulement sont de langue spécialisée ou de spécialité. Le premier en date est un dictionnaire de phraséologie de Giuseppe Bourelly (Frasi, espressioni figurate o metaforiche) de 1839. En 1870 est édité le dictionnaire de termes de marine de Luigi Fincati. Ce centre d'intérêt se poursuit au XX ${ }^{\mathrm{e}}$ siècle (Americo Bertuccioli en 1916 et 1939) et s'élargit en 1925 avec la publication du Dictionnaire illustré de l'aéronautique, toujours de Bertuccioli. La Première Guerre a produit un poche «à l'usage des infirmières ». A partir de la seconde moitié du siècle, les préoccupations sont plus d'ordre commercial et technique (Bianchi et Dubois en 1956, ...) et encore militaire (Oldra A.O. pour les termes militaires en 1962) (voir Appendice). Nous ne trouvons plus de dictionnaires de langue spécialisée ou de spécialité après cette date. Il est à remarquer qu'il ne s'agit pas de réduction de dictionnaires plus grands.

\section{2}

Les événements de politique extérieure ou scolaire, la vision qu'ont les deux nations l'une de l'autre à un moment donné de l'histoire influencent positivement ou négativement la diffusion des dictionnaires. Le premier moment de grande diffusion de ces bilingues de poche se situe pendant la Révolution française et le premier Empire napoléonien qui, au début, a été accueilli avec enthousiasme en Italie. Le deuxième commence dans les années 1830 et dure pendant toute la Monarchie de juillet, qui, du point de vue scolaire, voit l'approbation de la loi Guizot (1833), mais celle-ci ne concerne que l'école élémentaire et ne prend pas en compte l'enseignement des langues étrangères. D'autre part, dans l'Italie encore désunie, rien ne justifie cette flambée, si ce n'est un engouement diffus pour la langue et la culture françaises dans toute la péninsule et une recrudescence des échanges commerciaux. Cette phase culmine ${ }^{8}$ pendant la première décade du second Empire napoléonien, juste avant l'unification italienne de 1860 (et l'on sait le rôle joué par la France à cette occasion). Les pics suivants se situent pendant la Première Guerre mondiale, qui voit la France et l'Italie alliées contre l'ennemi commun, et dans les années 60 et 90 du $\mathrm{XX}^{\mathrm{e}}$ siècle. Les années 60 correspondent non seulement à la période gaullienne et à la mise en train de la Communauté Economique Européenne ${ }^{9}$ mais aussi à l'entrée en vigueur en Italie de la loi du 31 décembre 1962, qui crée le collège unique (Scuola media unificata) et qui renouvelle l'enseignement des langues modernes. Dans les années 90, que ce soit en France ou en Italie, on voit prendre des initiatives au niveau gouvernemental pour ce qui est du renforcement de l'apprentissage des langues vivantes même au niveau élémentaire. Malheureusement cela signifie le plus souvent surtout enseignement de l'anglais. Ce pic $^{10}$ est donc sans doute dû non seulement aux échanges touristiques intenses entre les deux nations mais aussi à l'utilisation de plus en plus diffuse de cette typologie de dictionnaires dans les établissements d'enseignement à un moment où les bilingues scolaires (Candido Ghiotti, Augusto Caricati, ...), plus gros et donc plus chers, se vendent de moins en moins (Lillo 2006 : 228 ) (Fig. 1). 


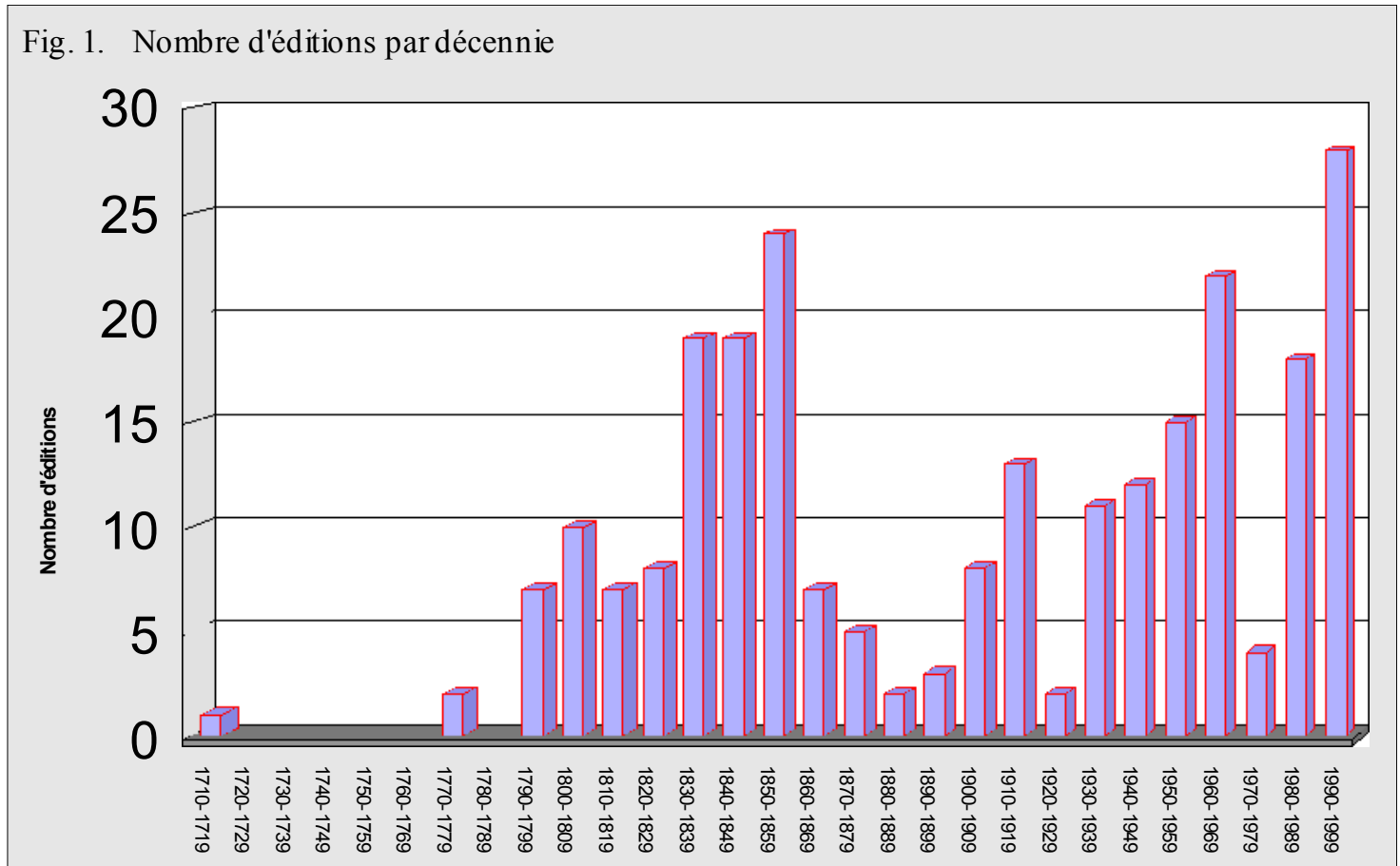

\section{3}

De tous les dictionnaires retrouvés d'un même auteur, bien peu sont de nouvelles éditions et beaucoup des réimpressions. Il est bien connu que, sous des dehors trompeurs (nouvelle édition, revue et corrigée, ...), les maisons d'édition reproposent tels quels, ou avec des modifications minimes, des textes déjà publiés. Les poches ne font évidemment pas exception. Pour ne citer que quelques exemples : Hatier publie en 1916, dans la collection Poucet, le texte de Faustina de Graziani qu'il fait ressortir sous la même forme cinquante deux ans après (1958) en éliminant uniquement un des regroupements thématiques de la fin, relatif aux termes militaires (la macrostructure est absolument identique), de même Magnard reproduit en 1980 le même ouvrage qu'en 1955, donnant, dans ses pages grises, exactement la même liste de néologismes que vingt-cinq ans auparavant (et il est clair qu'alors insucesso n'est plus perçu comme néologisme).

\section{Les auteurs (voir Appendice)}

\section{1}

Au XVIII ${ }^{\mathrm{e}}$ siècle, le célèbre dictionnaire d'Alberti di Villanova est abrégé. Au XIX ${ }^{\mathrm{e}}$, les auteurs de poches les plus prisés sont Giuseppe Martinelli, Jacques-Louis-Barthélemy Cormon / Vincenzo Manni, A. Briccolani, Antonio Ronna seul ou avec Giuseppe Filippo Barberi ou G. Biagioli ${ }^{11}$ et Marie-Jean Blanc Saint-Hilaire, qui s'inspire du Cormon et Manni ; un nombre important de dictionnaires est publié anonymement (dans les années 40 et $50 \mathrm{du} \mathrm{XIX}^{\mathrm{e}}$ siècle, les noms de Cormon et Manni sont utilisés dans les titres pour attirer l'acheteur). $\mathrm{Au} \mathrm{XX} \mathrm{X}^{\mathrm{e}}$, Cesare Bergoglio, Arturo Angeli avec ou sans Costanzo Ferrari, Carlo Boselli, Giuseppe Padovani avec ou sans Richard Silvestri (Larousse), L. Marichy et Ettore Zelioli / François Barucchello / Giovanna Ferraguti (Collins) ont le plus gros tirage. Pendant la deuxième moitié de ce siècle, le nombre d'auteurs augmente en même temps que diminue la quantité de 
dictionnaires publiés par chacun d'eux. Les anonymes sont aussi nombreux qu'au siècle précédent (Voir Appendice).

\section{2}

Jusqu'au début du $\mathrm{XX}^{\mathrm{e}}$, tous les lexicographes sont des hommes. La première femme à proposer un poche est Faustina de Graziani qui publie en pleine Première Guerre mondiale $(1916,1917)$, ce qui met encore une fois en évidence le rôle de la Grande Guerre dans le processus de libération de la femme. Nous avons ensuite Mlle Marichy (c'est ainsi qu'elle se présente) en 1940 (son dictionnaire sera publié jusqu'en 1960), et plus tard Paola Banfichi Ferrari et Adriana Secondo pour les Collins Gem (1964, 1983). A partir des années quatre-vingt, elles sont très nombreuses dans les comités de rédaction des dictionnaires contemporains.

\section{3}

Les années $60 \mathrm{du} \mathrm{XX}^{\mathrm{e}}$ siècle sont très importantes parce que l'on voit la maison d'édition se propulser au premier plan à côté du nom de l'auteur, qu'elle efface même parfois complètement. Bien vite, on n'appelle plus un dictionnaire par le nom de son auteur mais par celui de la maison d'édition.

\section{4}

Dans les années 80, une société de services éditoriaux, Edigeo, commence à publier des dictionnaires sur commande. Elle emploie un réseau important de collaborateurs, traducteurs, terminologues, professionnels, qui restent anonymes. Elle collabore avec les plus grandes maisons d'édition (Rizzoli Larousse, Zanichelli, Hoepli, ...).

\section{Pourquoi publier des poches?}

\section{1}

Avant tout, comme nous l'avons vu dans la définition, pour proposer au public un instrument moins encombrant, plus maniable et surtout moins lourd.

\section{2}

Ensuite, pour réduire au minimum le prix d'achat de ces dictionnaires. Au XIX ${ }^{\mathrm{e}}$ siècle, comme nous venons de le dire, Briccolani, Barberi / Ronna, Ruggieri offrent au public des ouvrages de dimensions extérieures très réduites mais qui, du point de vue du contenu, sont beaucoup plus proches des dictionnaires de format entier. Ils proposent en effet au lecteur un très grand nombre de pages (Ruggieri et Gérard, s.d., a 1115 pages !) ${ }^{12}$ et une quantité très importante d'entrées grâce à l'utilisation de caractères typographiques extrêmement petits (56 lignes pour une justification de $11,5 \mathrm{~cm}$ chez Briccolani, 1831, Paris, Thiériot, ou 67 pour une justification de 9,5 cm chez Barberi / Ronna, Edition Diamant, 1843, Paris, Baudry). Cela dans le but de présenter l'ouvrage le plus complet et le moins cher possible. Martinelli exprime ces mêmes préoccupations dans sa préface ${ }^{13}$ et Cormon et Manni aussi veulent faire meilleur marché qu'Antonini, Veneroni ou Alberti.

\section{3}

A toutes les époques cette préoccupation va de pair avec l'extension de la scolarisation qui rend nécessaire la publication d'instruments de travail à des prix abordables (Il piccolo Ghiotti,...). Comme nous l'avons vu précédemment, le dictionnaire de poche est donc souvent synonyme de dictionnaire 
pédagogique. Ce format réduit, avec en conséquence l'élimination de difficultés inutiles pour les débutants, facilite l'apprentissage ${ }^{14}$.

\section{4}

Depuis toujours il est emporté par les voyageurs ${ }^{15}$ lors de leurs pérégrinations et aujourd'hui par les touristes.

\section{5}

D'autre part, de façon plus surprenante, ils sont utiles aux «personnes qui cultivent la littérature », au $\mathrm{XIX}^{\mathrm{e}}$ comme au $\mathrm{XX}^{\mathrm{e}}$ siècle. Briccolani n'hésite pas à donner des exemples littéraires, mais aussi les Moussard et Garrus ou les Garzanti ont comme objectif déclaré l'apprentissage de la langue littéraire.

\section{6}

Dans les années 1980 apparaît un phénomène intéressant : des dictionnaires de poche sont offerts en suppléments gratuits d'un autre produit, dans le but de pousser le consommateur à l'achat: en 1886 et 1992 par l'hebdomadaire Espresso (dans son n ${ }^{\circ} 8$ du 2 mars 1986 et nº 10 de mars 1992), en 1988 (n ${ }^{\circ}$ 1174 du 10/10/1988) par la revue Panorama, en 1994 par le quotidien Il Corrriere della Sera ${ }^{16} \ldots$.. En 1993, le De Agostini est proposé en même temps que la revue Il fisco, en 1996 Edigeo publie chez Zanichelli (Bologne) une édition spéciale hors commerce pour Kodak et, en 1998, pour un fabricant de riz (le Riso Gallo).

\section{Les dictionnaires de poche sont-ils des abrégés de dictionnaires plein format?}

\section{1}

Cette affirmation est valable pour le portatif de $1775^{17}$ et les abrégés / portatifs d'Alberti (XVIII et XIX ${ }^{\mathrm{e}}$, mais n'est pas applicable à l'ensemble des dictionnaires bilingues de poche (ou portatifs) du XIX, comme cela est communément accepté ${ }^{18}$. Martinelli, Cormon et Manni, Briccolani, Lauri, Ruggieri et Gérard, Tauchnitz, Aquenza, n'ont publié que des dictionnaires de poche pendant tout le siècle. Seul Barberi est d'abord sorti en format de poche (1822) puis s'est transformé en Grand dictionnaire [...] en 1838. De même, en 1860, bien après la première édition de Briccolani, Da Fonseca et Briccolani publient un dictionnaire plein format. Cette règle se maintient pendant toute la première moitié du XX. . En effet Boselli, Le Boucher, Lucifero Darchini ${ }^{19}$, Fuccini, Marichy ne sont présents sur le marché qu'en format «poche » avec, cependant, l'exception de Bergoglio qui, avant la publication de ses poches, présente au public en 1890 un dictionnaire (Vocabolario italiano-francese, letterario, scientifico, commerciale, ...), qui est, de toute façon, au format poche $(11,5 \mathrm{~cm} \times 17,5 \mathrm{~cm} \times 7 \mathrm{~cm})$.

\section{2}

Cette pratique s'inverse à partir de la seconde moitié du $X X^{\mathrm{e}}$.siècle. Des valeurs sûres, tels que Ghiotti, Mariotti, déjà connus et appréciés sur le marché comme auteurs de dictionnaires plein format, sont proposés au public en poche: le Piccolo Ghiotti, en $1949^{20}$, le Piccolo Mariotti ${ }^{21}$ en 1951. Le dictionnaire de Lucifero Darchini, qui n'a jamais été qu'un poche, devient le Piccolo Darchini (1961), sans doute comme opération de marketing tendant à s'approprier de la célébrité du père Gaetano Darchini ${ }^{22}$. Seuls Moussard / Garrus (1955-1956) et Balmas (Piccolo Balmas, 1971) publient d'abord en poche puis continuent avec des dictionnaires plein format (Moussard et Garrus en 1980-1982, Balmas en 1975). Balmas reprend le filon dans les années $90(1993,1998)$ en publiant son Mini dictionnaire. 


\section{Quels sont les critères de sélection des entrées ?}

L'éventail est très large. Le lexicographe veut, avant tout, donner l'usage commun de la langue ${ }^{23}$, refusant explicitement les termes désuets (Boselli). Selon les époques, ou les modes, il propose aussi des termes spécialisés, par exemple : tous les termes propres des sciences et des arts ${ }^{24}$ (Martinelli) ou la langue des nouvelles technologies (Ferrari et Angeli, Boselli, Garzanti...), de la finance, de l'économie, de la gestion, du sport, de la bureautique (Garzanti / Le livre de poche, 1984, 1994), etc. De plus, comme ces dictionnaires sont, comme nous l'avons dit, de par leur format et leur prix, des manuels scolaires, il en résulte, notamment au XIX ${ }^{\mathrm{e}}$ siècle, une épuration des termes bas ou vulgaires Enfin, après les études de Gougenheim, le français fondamental sert de modèle (Noaro 1993, Collins Mondadori,...) mais, aux antipodes, on a encore des lexicographes qui maintiennent le lexique littéraire pour faciliter la compréhension des textes des grands auteurs (Moussard et Garrus, Garzanti / Livre de poche).

\section{Economiser de l'espace est une nécessité.}

Les mécanismes adoptés pour économiser de l'espace varient d'un auteur à l'autre, ou d'un éditeur à l'autre.

\section{1}

Nous avons déjà commenté l'utilisation de caractères typographiques extrêmement petits (Briccolani, Barberi et Ronna). On peut ajouter à cela l'élimination des majuscules dans les entrées (Boselli), ou le fait d'envahir l'espace laissé libre dans l'entrée précédente ou suivante afin de réduire le nombre de lignes et donc de pages.

\section{2}

Padovani (première édition 1930) procède par regroupement morphologique. Il ne donne in extenso que l'entrée principale. Les dérivés sont présentés à la suite, amputés de la partie commune, qui ne correspond pas forcément à la racine. Cette partie est représentée par un tiret : « conv $\|$ aincant, ante [...].

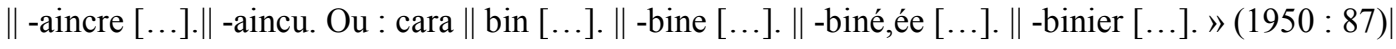

\section{3}

Quant au paratexte, il est soit absent, soit extrêmement restreint. Il n'est pas rare que ces dictionnaires soient dépourvus de préface. Quand les notions grammaticales sont présentes, elles se limitent aux conjugaisons des verbes (et quelquefois aux nombres). En revanche, les dictionnaires de dernière génération sont un peu plus prolixes et expliquent volontiers «comment utiliser ce dictionnaire » (Larousse, Robert). Les noms géographiques, historiques, mythologiques, les noms propres de personnes, de nations anciennes et modernes sont toujours présents au $\mathrm{XIX}^{\mathrm{e}}$ siècle, le plus souvent sous forme de liste(s) autonome(s), à côté de la macrostructure. $\mathrm{Au} \mathrm{XX}$, ils y sont parfois insérés mais leur présence n'est plus systématique. On pourrait alléguer qu'il s'agit là d'une preuve évidente que ces ouvrages servent pour les voyageurs comme cela est parfois déclaré dans l'introduction. Le fait n'est évidemment pas exclu mais la présence des noms anciens des nations, des noms historiques ou mythologiques, confirme notre conviction qu'il s'agissait aussi, au XIX ${ }^{\mathrm{e}}$, d'ouvrages pour la compréhension de textes de culture. $\mathrm{Au} \mathrm{XX}^{\mathrm{e}}$ siècle, ces dictionnaires peuvent inclure des « modèles de conversation ». 


\section{4}

\subsection{1}

Pour ce qui est de la microstructure, on peut remarquer que la description de la prononciation est presque absente des dictionnaires du $\mathrm{XIX}^{\mathrm{e}}$ siècle $^{25}$ et de la plupart des ouvrages ${ }^{26}$ de la première moitié du XX (certains indiquent uniquement l'accentuation des mots). La transcription de la prononciation se généralise peu à peu après 1950 . L'API commence à être utilisé très tard dans le siècle. Au XIX ${ }^{\mathrm{e}}$, cela pourrait s'expliquer par le manque d'outils disponibles (comment décrire les nasales, le /y/, etc. ?) mais nous savons que, dans les grammaires pour l'enseignement du FLE à l'époque, les auteurs avaient déjà tant bien que mal décrit les sons du français. Il faut rechercher ailleurs la raison de ce phénomène. Il réside toujours dans le fait déjà mentionné au point précédent : les dictionnaires du XIX ${ }^{\mathrm{e}}$ siècle sont aussi utilisés comme dictionnaires généraux pour aider à la compréhension de textes (souvent littéraires) ${ }^{27}$ et à l'expression écrite ${ }^{28}$.

\subsection{2}

D'autre part, du point de vue morphologique, plus on s'approche du $\mathrm{XX}^{\mathrm{e}}$ ou du $\mathrm{XXI}^{\mathrm{e}}$ siècle, plus les auteurs ont tendance à faire disparaitre des entrées qui peuvent être considérées comme superflues : les participes passés des verbes (présents chez Cormon / Manni au XIX ${ }^{\mathfrak{e}}$ siècle), les adverbes en -ment lorsque leur construction est parfaitement régulière, parfois même des substantifs ou des adjectifs dont on peut déduire la formation d'une forme précédente : le Robert Collins 2005 donne condamner mais pas condamnation, vandalisme mais pas vandale...

\section{5}

Les dictionnaires de dernière génération présentent à l'intérieur de la nomenclature des encadrés grammaticaux qui veulent aider à résoudre les principaux problèmes liés à un « mot clé » (tutto, quale, $o \grave{u}, \ldots$ ) ou qui mettent en garde contre de faux amis (Robert Collins Gem, 2003). Ces encadrés deviennent encyclopédiques quand ils servent à élucider le sens de mots à très forte charge culturelle ou civilisationnelle (arrondissement, légion d'honneur, laurea,...). Dans ce dernier cas, la nécessité d'économiser de l'espace laisse place à la volonté de mettre en évidence la notion présentée et de rentabiliser au maximum l'espace à disposition

\section{Conclusion}

Le dictionnaire de poche se définit par rapport à d'autres dictionnaires de format plus grand. Au XIX ${ }^{\mathrm{e}}$ siècle le contenu linguistique peut être extrêmement varié et touffu. L'affirmation selon laquelle les poches seraient des abrégés de dictionnaires de format entier est irrecevable jusqu'en 1950 et discutable après cette date. Depuis la Révolution, les poches sont largement diffusés dans la population, grâce notamment à leur prix réduit et à leur emploi en milieu scolaire. A cause de la nécessité de brièveté, les objectifs et les contenus se sont modifiés suivant les époques et les publics. De nos jours, la valeur ajoutée à ces dictionnaires n'est plus littéraire mais contrastive et civilisationnelle.

\section{Références bibliographiques}

Bray, L. (1988). Notes sur la genèse des dictionnaires portatifs français. L'exemple du Dictionnaire portatif de la langue françoise, extrait du grand dictionnaire de Pierre Richelet, 1756. Travaux de linguistique et de philologie, XXVI, 95-111.

Gougenheim, G.; Michéa, R.; Rivenc, P.; Sauvageot, A. (1956). L'élaboration du français élémentaire. Etude sur l'établissement d'un vocabulaire et d'une grammaire de base. Paris : Didier.

Lillo, J. (2006). Les dictionnaires pédagogiques français / italien. Quaderni del CIRSIL, 5, 221-236. 
Lillo, J. éd. (2007). Quattro secoli di lessicografia italo-francese. Repertorio analitico di dizionari bilingui. Berna : Peter Lang.

Quemada, B. (1967). Les dictionnaires du français moderne 1539-1863. Etude sur leur histoire, leurs types et leurs méthodes. Paris : Didier.

Rey, A. (1989). Le français et les dictionnaires aujourd'hui. Recherches Applications, Août-septembre 1989, 78-83.

\section{Appendice des dictionnaires «Portatifs », "Abrégés », " Mini » ou « De Poche », " Portatili », « Piccoli », "Compendiati » ou « Tascabili » par ordre chronologique en fonction de la première édition.}

Toutes les données relatives à ces dictionnaires se trouvent dans Lillo J. éd. (2007)

Anguselli da Luca Biagio : 1710

Francesco Alberti Di Villanova : 1774-1775, 1792, 1793 (Genova, Franchelli), 1793 (Genova, Repetto), 1793 (Venezia, Bettinelli), 1798, 1804, 1804 (bis), 1810

Anonymes : 1775, 1803, 1806, 1812, 1828, 1833-1834, 1839 (selon Cormon et Manni), 1840 (selon Cormon et Manni), 1842 (selon Cormon et Manni), 1843 (selon Cormon et Manni), 1851 (selon Cormon et Manni), 1851 bis (selon Cormon et Manni), 1852 (selon Cormon et Manni), 1853 (selon Cormon et Manni), 1854 (selon Cormon et Manni), 1854 bis (selon Cormon et Manni), 1855 (selon Cormon et Manni), 1856 (selon Cormon et Manni), 1860 (selon Cormon et Manni), 1913 (à l'usage des infirmières), 1939, 1944, 1959 (?), 1960 (Langenscheidt), 1960 bis (Langenscheidt), 1960 (Malipiero), 1960 (Martello), 1961 (Dictionnaire Lilliput..., Larousse), 1964, 1969, 1973, 1975, 1987, 1995, 1996, 2000, 2002, s.d. (Levasseur)

Eiraud Antoine : 1797

Martinelli Giuseppe : 1797, 1801, 1803, 1807, 1819, 1843

Cormon Jacques-Louis-Barthélemy / Manni Vincenzo : 1802, 1807, 1808-1809，1813，1823，1836, $1838,1843,1847,1850,1851,1854,1856,1857,1865,1874$

Lauri Ange/Angelo : 1810, 1819, 1832, 1833

Hamonière G. : 1819

Barberi Giuseppe Filippo : 1822, 1826

Chapellon Carlo, Cormon Jacques-Louis-Barthélemy, Manni Vincenzo : 1823. 1823 bis

Catineau : 1826

Briccolani A. : 1830, 1831, 1833, 1834, 1836, 1837, 1842, 1843, 1850, 1855, 1866, 1874

Bianchi E. : 1832

Barberi Giuseppe Filippo, Ronna Antonio : 1836, 1842, 1843, 1847, 1850

Ronna Antonio, Biagioli G. : 1836, 1836 bis, 1838, 1839-1840, 1840, 1841, 1841 bis, 1846, 1848, 1851, 1855,1859

Bourelly Giuseppe : 1839 (Frasi, espressioni figurate o metaforiche...)

Ruggieri Giuseppe, Gérard F.G. : 1840, 1881, s.d., s.d. bis

Blanc Saint-Hilaire Marie-Jean : 1846, 1849, 1851, 1851 (bis), 1854, s.d.

Lauri Angelo / Saint-Ange de Virgile : 1858

Ronna Antonio : 1858, 1861, 1864, 1883, s.d., s.d. 
Briccolani A., Da Fonseca : 1860

Ferrari Costanzo : 1867, s.d.

Fincati Luigi : 1870 (Termes de marine)

Tauchnitz Charles : 1871, 1893

Galpinozzi Ciro : 1875

Aquenza Giuseppe : 1898, s.d.

Bergoglio Cesare : 1899, 1910, 1912, 1914, 1932, 1935, 1941-1942, 1946

Angeli Arturo : 1900, 1903, 1908, 1912, 1928-1929, 1935, 1941, 1949, s.d.

Ferrari Costanzo, Angeli Arturo : 1900, 1904, 1909, s.d. (1918), s.d.

Boselli Carlo : 1902, 1909, 1910-1914, 1930, 1944, 1947, s.d., s.d. bis

Le Boucher Gaston : 1911, 1912

Josso M.G. : 1915, 1917, 1960

Bertuccioli Americo : 1916 (termes techniques et de marine) ; 1925 (Dict. illustré de l'aéronautique) ; 1939 (Dict. de marine)

De Graziani Faustina : 1916, 1917, 1958

Ferrari Costanzo, Angeli Arturo, Michel L. : 1934

Fuccini Pietro : 1934

Genty Paul : 1934

Padovani Giuseppe : 1930, 1935, 1941, 1950

Marichy L. : 1940, 1947, 1951, 1960, s.d.

Darchini Lucifero : 1946, 1961 (Il piccolo Darchini)

Ghiotti Candido, Chanoux Antonio (Il piccolo Ghiotti) : 1949, 1956, 1960, 1973

Del Guerra Elena : 1951

Mariotti Scevola (Il Piccolo Mariotti) : 1951, 1957

Moussard J., Garrus R. : 1955-1956, 1980, 1982

Bianchi P., Dubois A. : (Piccolo dizionario commerciale) 1956

De Vercellis Anton Sandro (Il piccolo De Vercellis) : 1958, 1964

Gioseffi Guido (Piccolo dizionario ... commerciale...) : 1958

Platy Vincenzo (Petit dictionnaire... technique) : 1958

Ropa A., Dubois A. (Piccolo dizionario tecnico della lavorazione dei metalli ...) : 1958

Salvi Lia : 1958, 1985

Laurent G. : 1960, 1967, 1983, 1987

Rostan Edwin : 1960

Koller Adelaide : 1961

Oldra Armand (Termes militaires) : 1962 
Banfichi Ferrari Paola, Secondo Adriana (Collins Gem) : 1964, 1981, 1983

Bloncourt-Herselin Jacqueline : 1964, 1968, 1986

Padovani Giuseppe, Silvestri Richard : 1964, 1989, 1992

Pelissier Jean : 1966

Balmas Enea : 1971 (Il piccolo Balmas), 1972 (Il piccolo Balmas), 1993 (Mini dictionnaire), 1998 (Mini dictionnaire)

Christie Barbara, Macginn Maìri, Zelioli Ettore (Collins Mondadori) : 1981

Zelioli / Baruchello / Ferraguti : 1984 (Collins/Marabout), 1984 (Collins Mondadori, Hachette), 1985, 1990, 1990 bis, 1993, 1995, 1996, 1998, 1998 bis, 1999

La Tour Filippi : 1987, 1991, 1994, 1999

Balmas / Bocassini (Hachette \& De Agostini) : 1989. 1993 (Il fisco), 1999, 2003, 2005

(Edigeo) : 1986 (L’Espresso Dizionario per l'Europa), 1989, 1992 (L'Espresso Dizionario per l'Europa), 1993, 1996 (pour Kodak), 1998 (pour Riso Gallo)

Noaro Pierre, Cifarelli Paolo, Nora Galli De’ Pratesi : 1993

Perhauz Maria-Sofia, Trifari Valeria : 1994, s.d. (Corriere della Sera)

Robert / Collins (Michela Clari) : 1994, 1999, 2003, 2005

Mini Dictionnaire Larousse : 1999, 2005

Dictionnaire de poche Larousse : 1999, 2003, 2004, 2005

Garzantini : 2000

Asti Giuseppe, Ferrario Oreste, Cormon Jacques-Louis Barthélemy, Manni Vincenzo : s.d., s.d. bis Lombardo G.M. : s.d.

Luppi M., Cormon Jacques-Louis Barthélemy, Manni Vincenzo : s.d.

Ronna A. : s.d. (1858), s.d., s.d. bis

Ruggieri Giuseppe : s.d.

1 La rédaction d'un répertoire analytique de tous les dictionnaires bilingues français / italien retrouvés dans presque quatre cents bibliothèques en Italie et à l'étranger (Lillo éd. 2007) permet d'avoir une vision très large sur la question traitée.

2 (1737-1801). On trouve indifféremment pour cet auteur les noms suivants : Francesco Alberti, Francesco De Alberti di Villanova, Francesco D'Alberti Di Villanova, Francesco Alberti di Villanova, Francesco Alberti di Villanuova et, comme il est coutume à l'époque, François Alberti, François Alberti de Villeneuve, François d'Alberti de Villeneuve. Nous donnons les dates de naissance et de mort des lexicographes quand elles nous sont connues. Nous ne les possédons pas pour la plupart d'entre eux.

3 Jacques-Louis-Barthélemy Cormon (17..-18..).

$4 \quad$ L'Appendice à la fin du texte donne tous les prénoms connus des lexicographes.

5 (1564-1609).

6 Un italien-français, un italien-allemand et un latin-italien.

7 Jean Vigneron dit Giovanni Veneroni ou le Sieur de Veneroni (1642-1708).

$8 \quad$ Six éditions en 1851, l'année du coup d'état de Louis-Napoléon Bonaparte. 
$9 \quad$ Six éditions en 1958 et neuf en 1960 !

10 Trente éditions.

11 Giosafatte Nicolò Biagioli est aussi auteur d'une grammaire française pour italophones, Grammatica ragionata della lingua francese, Milan, Silvestri, 1827 ( $2^{\mathrm{e}}$ édition).

12 Bray (1988: 101, note 15) signale le même phénomène à propos d'un dictionnaire français-allemand du XVIII .

13 «Un dictionnaire d'une forme aussi portative ne peut être que très-commode aux personnes occupées de l'une de ces langues, par la facilité qu'il leur présente d'avoir constamment sur eux le moyen d'éclaircir sur-le-champ les doutes qu'une lecture ou une conversation pourrait leur faire naître. Les écoles trouveront également dans la modicité de son prix, l'avantage de remplacer les grands dictionnaires que la cherté ne leur permet pas de se procurer $\gg(1807$, vii-viii).

14 Martinelli affirme que «L'on pourrait même avancer que ces livres volumineux (les D'Alberti) sont communément plus nuisibles qu'utiles aux commençans, en ce qu'ils leur font perdre beaucoup de temps en fixant leur attention sur des objets hors de leur portée, et qui les écartent de leur but principal » (Ibid. : viii). Briccolani publie aussi «pour la jeunesse ».

15 «A l'usage des voyageurs et des écoles» (Aquenza, 1898). «Il Piccolo Ghiotti è un vademecum [...] non solo nelle tasche dello studente ma anche di chi viaggia, per sport, per affari ».

$16 \quad$ La date de parution du journal n'est pas mentionnée dans le dictionnaire : Supplemento gratuito al Corriere della Sera odierno (Supplément gratuit au Corriere della Sera de ce jour).

$17 \quad$ L'Esprit des langues françoise et italienne, ou Dictionnaire abrégé de l'Académie de la Crusca, à l'usage des personnes qui veulent parler et écrire correctement ces deux langues. Paris, Gostard. Il ne figure pas dans le répertoire (Lillo éd. 2007).

18 «Les abrégés issus par filiation plus ou moins directe de répertoires de type extensif» (Quemada 1967: $250)$; «Ces dictionnaires portatifs ou de poche sont des abrégés » (Rey $1989: 8$ ).

19 Lucifero Darchini est le fils de Gaetano. Le père a rédigé un dictionnaire scolaire, le fils uniquement des poches.

20 Ghiotti commence à publier en 1883 son dizionario comparativo et en 1892 son dizionario scolastico.

21 La première édition du dictionnaire général de Scevola Mariotti est de 1942.

22 La première édition du dictionnaire de Gaetano Darchini est de 1902.

23 Il Piccolo Ghiotti veut introduire «le parole comune della lingua parlata e della lingua viva ». Larousse $(1949,1989)$ veut satisfaire «aux besoins de la vie courante », Noaro, 1993, s'inspire à la langue «des médias, journaux, radio, télévision » ...

24 L'influence de l'Encyclopédie de Diderot et D'Alembert et des dictionnaires de Francesco D'Alberti di Villanova se fait sentir.

25 Seuls Cormon et Manni proposent une transcription basée sur la valeur phonique des différentes lettres de l'alphabet.

26 Gaston Le Boucher propose sa méthode Toussaint-Langenscheidt.

27 On peut citer à ce propos la préface de l'édition de 1866 de Briccolani : « Le dictionnaire [...] du chevalier Briccolani avait besoin d'être augmenté d'un grand nombre de mots, de phrases et locutions qui se trouvent dans les classiques des deux nations. J'ai donc pris sur moi la tâche longue et difficile de lire et méditer avec soin ces mêmes classiques en confrontant les originaux avec les versions : cette lecture m'a fourni une foule d'exemples qui font connaître le génie des deux langues, exemples qui ne se trouvent point dans les dictionnaires Français-Italien et Italien-Français publiés jusqu'à ce jour. Ces exemples n'ont point été pris au hasard. Une attention scrupuleuse a présidé à leur choix. Cet ouvrage, enrichi d'une infinité de termes de sciences, d'arts et de métiers, d'expressions accréditées par l'usage et consacrées par les bons écrivains de l'époque, pourra être très utile à l'enseignement de la jeunesse, et même à toutes les personnes qui cultivent la littérature »([6]) (C'est nous qui soulignons) 
DOI $10.1051 / \mathrm{cmlf08246}$

28 La tâche qu'il nous restait à remplir pour perfectionner la troisième édition que nous publions [...] était [...] de l'enrichir de quelques centaines de mots nécessaires à ceux qui veulent écrire correctement le bon toscan, ainsi que de diverses locutions employées fréquemment par ceux qui, Toscans ou non, ont cependant écrit cette langue avec autant de grâce que d'élégance (Martinelli, 1807 : 5, Avis des éditeurs). 